\title{
ALGUNAS REFLEXIONES SOBRE LA MIGRACIÓN ALTAMENTE CUALIFICADA: POLÍTICAS, MERCADOS LABORALES Y RESTRICCIONES ${ }^{1}$ SOME REFLECTIONS ON HIGHLY SKILLED MIGRATION: POLICIES, LABOUR MARKETS AND CONSTRAINTS
}

\author{
Beatriz Padilla \\ ISCTE - Instituto Universitario de Lisboa, \\ CIES-IUL, Lisboa, Portugal. \\ beatriz.padilla@iscte.pt \\ Recibido: 8/10/2010 \\ Aceptado: 10/12/2010
}

\begin{abstract}
Resumen
El presente artículo tiene por objeto reflexionar sobre la migración altamente cualificada, teniendo en cuenta los países de origen y de destino, empleando contribuciones de la literatura internacional, principalmente de Latinoamérica, y examinando ejemplos del Sur de Europa. Estas reflexiones han inspirado algunas recomendaciones sobre políticas para mejorar el papel de las diásporas con talento.
\end{abstract}

Palabras clave: migración altamente cualificada; talento internacional; diásporas

Abstract

The aim of this article is to reflect on highly skilled migration accounting for countries of origin and destination, using contributions from the international literature, mainly the one produced in Latin America, and focusing on examples from Southern Europe. These reflections inspire some policy recommendations to enhance the role of talented diasporas.

Keywords: highly skilled migration; international talent, diasporas

\footnotetext{
${ }^{1}$ Presentado en el Seminario Internacional Migraciones Circulares, el retorno como estrategia de desarrollo en los países de Origen, celebrado en Alicante, del 6 al 8 de octubre de 2010, organizado por el Instituto Interuniversitario de Desarrollo Social y Paz, con la colaboración del Ministerio de Ciencia e Innovación (Ref. CSO2009-07331-E) y de la Conselleria d'Educació de la Generalitat Valenciana (Ref. AORG/2010/124)
} 


\section{INTRODUCCIÓN: EL PROBLEMA DE LA MIGRACIÓN Y LA GLOBALIZACIÓN}

La migración siempre ha acaparado la atención de forma intermitente, pero con la globalización la movilidad ha pasado a ser uno de los fenómenos más destacados. Lo cierto es que, como han señalado muchos especialistas (Solimano, 2006; Sassen, 1998 y 1999; Mittlelman, 1997; Hirst y Thompson, 1996; Ruiz Sandoval, 2007), la globalización se ha centrado en su mayor parte en la movilidad internacional de bienes y capital, dos de los factores de producción, y no tanto en la movilidad de las personas, formadas o no, que constituyen el otro factor clave.

En la actualidad, se presta cada vez más atención a la migración. En este sentido, en términos extremos, los migrantes son percibidos, principalmente, como los que tienen brazos y manos (denominados comúnmente "migración laboral" o "económica") o como los que tienen cerebro (denominados comúnmente "migración de talentos", "migración altamente cualificada" o "fuga" o "ganancia de cerebros" según la situación geográfica). La tendencia general consiste en considerar que los que pertenecen a la primera categoría son necesarios, mientras que los de la segunda son deseados. Muchos son los factores que han contribuido a la distinta evaluación y valoración de la situación de los migrantes, pero es indudable que la "aparición y la expansión de la llamada 'sociedad del conocimiento' y su infraestructura tecnológica han agravado la preocupación por obtener un suministro adecuado de trabajadores altamente cualificados en los diversos países" (Barrere et al., 2004), otorgando más valor a los que cuentan con titulaciones y talento. Además, la movilidad internacional de los trabajadores altamente cualificados plantea desafíos y oportunidades al mundo en desarrollo, según D'Costa, que afirma lo siguiente:

Aunque los mercados laborales para los trabajadores capacitados no están tan globalizados como los mercados de capital (Brown 2001: 26-9), las pruebas, cada vez más numerosas, de la movilidad internacional del talento técnico (Docquier y Rapoport, 2004) han suscitado numerosas preguntas sobre las causas y las consecuencias de dicho movimiento, tanto para los países emisores como para los receptores (2006: 1).

Así, no es casualidad que últimamente la cuestión de la movilidad de las personas haya pasado, de manera contundente, a formar parte de la agenda política de los países del Norte y del Sur, respecto a las mismas personas pero con intereses distintos (Mármora, 2002). La migración está sujeta al capricho de la moda, que la convierte en un fenómeno de moda positivo a veces y negativo en otras ocasiones. En este sentido, es posible encontrar en todas partes 
defensores y detractores de la migración, que normalmente dependen de los ciclos económicos, los mercados laborales y el clima político de cada país, entre otros factores. Mientras que algunos países de la Unión Europea se han declarado abiertos a la migración porque trae diversidad y una población joven (lo cierto es que las naciones europeas están envejeciendo con pocas esperanzas de poder sostener sus sistemas de pensiones en el futuro), otros la han demonizado con el argumento de que divide la sociedad al traer a personas de culturas y religiones opuestas a las tradiciones, las creencias y los valores europeos. Las organizaciones internacionales también han "descubierto" la migración y muchas dedican tiempo e informes a analizar el fenómeno y proponer todo tipo de recomendaciones (Naciones Unidas y sus agencias, el Banco Mundial y el FMI). Con todo, el mundo es testigo de la falta de gobernabilidad de la migración, lo que significa que la migración es a la vez funcional y disfuncional y que los gobiernos no han proporcionado "respuestas sociopolíticas adecuadas" a este tipo de cuestiones (Mármora, 2002).

La actitud ante la migración también está cambiando. Si nos fijamos sólo en la UE, podemos ver cómo países de gran tradición inmigrante, reconocidos como abiertos, progresistas y humanistas, como Suecia, Países Bajos y Francia entre otros, han adoptado nuevos marcos que no sólo restringen la migración, sino que también dificultan la integración y la adquisición de la nacionalidad y, además, defienden la deportación (Acosta, 2009). Por otro lado, países de tradición emigrante, como España y Portugal, han pasado a ser países de inmigración al abrir sus puertas a los recién llegados mientras su economía estaba en auge (Masanet y Padilla, 2010). Sin embargo, con la actual crisis económica, estos países se han visto obligados a cambiar sus políticas y han tenido que establecer otros tipos de medidas, sobre todo incentivos para el retorno, ya sea mediante los programas de retorno de la Organización Internacional para las Migraciones o convenciendo a los migrantes de juntar todas sus prestaciones por desempleo para regresar a su país de origen, con la esperanza de que se hayan resuelto los motivos que les hicieron partir al principio. Por lo tanto, la migración se convierte en un dilema sobre transferir el "problema de los migrantes" de un país a otro (véase la figura 1).

Dicho de otro modo, la migración abarca situaciones que se mueven a lo largo de un espectro continuo que va desde el problema de la migración y sus distintos puntos de vista (los "otros" que vienen a las sociedades de acogida, los que dejan su país natal llevándose sus capacitaciones consigo, la mano de obra no asimilada, la amenaza a la civilización occidental, etc.) hasta la solución de la migración y sus distintas posturas (las remesas que envían a su 
FIGURA 1

El espectro continuo de la migración como problema-solución

\section{Espectro continuo de la migración}

El "otro"

Traidor

Ladrón de trabajos

Utilizador de servicios sociales
Necesidad de mano de obra

Remesas

Factor demográfico

Contribuyente (pensiones, impuestos)
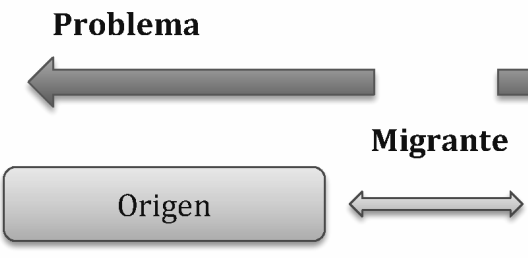

Destino

casa, la mano de obra, con o sin titulaciones, necesaria para cubrir una escasez en los recursos humanos, la contribución al sistema de pensiones de la UE y a la demografía). Así pues, siempre hay dos caras: un inmigrante es siempre un emigrante. Probablemente éste constituya uno de los enigmas no resueltos cuando se trata la migración, ya que por lo general las investigaciones o la elaboración de políticas sólo toman en consideración una cara, una perspectiva o un papel. En los casos en los que se produce una negociación sobre la migración, suelen participar Estados que no velan necesariamente por los intereses de los migrantes. En este sentido, Ruiz Sandoval ha tildado esta relación entre los países de origen y de destino de "diálogo de sordos" (2007: 170). Se suele percibir a los migrantes únicamente como mano de obra móvil y no como seres humanos con intereses, familia, necesidades y, por supuesto, expectativas.

El presente artículo pretende exponer algunas cuestiones relativas a la movilidad del capital humano altamente cualificado, concediendo mayor protagonismo a la literatura procedente de países de Latinoamérica, menos conocida en el Norte, y a la vez cuestionando el concepto de migración circular. Asimismo, mediante el empleo de algunas experiencias reales de la movilidad del talento internacional (sobre todo del sector sanitario, pero no exclusivamente), el ensayo propone recomendaciones para las políticas en este ámbito. 


\section{HACIA LAS MIGRACIONES TEMPORALES: SIN APRENDER DE LA EXPERIENCIA}

Mármora (2002) nos explica que, hoy en día, las migraciones se distinguen de las anteriores en calidad. Esto se debe a que, en la actualidad, la brecha entre la riqueza y la pobreza se está haciendo más profunda en todo el mundo. La migración de finales del siglo XIX y principios del XX obedecía a una redistribución de los recursos humanos desde las regiones demasiado pobladas hacia las regiones con escasa población, lo cual provocaba una redistribución de la mano de obra en una época en la que se contemplaba los recursos humanos como la riqueza de las naciones. Los italianos, españoles y portugueses, entre otros, que migraron a las Américas, lo hicieron como valores para el país de destino (para asentarse, trabajar, poblar y ocupar los territorios), aun cuando la población autóctona expresó resistencia.

Por otro lado, los movimientos de personas en el siglo XXI están revestidos de una filosofía distinta. La migración es la consecuencia de la brecha entre los ricos y los pobres en el mundo, de modo que se considera a la migración como la redistribución de la pobreza en un mundo caracterizado por la concentración de la riqueza, mientras que, al mismo tiempo, las personas, los migrantes, ya no representan la riqueza, a excepción de los migrantes con talento (Mármora, 2002). Además, la alteridad parece ser una característica específica de la migración, independientemente del estatus socioeconómico. Por lo tanto, los principales objetivos de las políticas migratorias actuales son controlar, regular y restringir la migración, con algunas excepciones, principalmente cuando los países o los sectores económicos sufren escasez de mano de obra (es decir, la construcción, el sector sanitario, el cuidado personal y doméstico, etc.).

En consecuencia, el migrante ideal hoy es el migrante temporal, que regresa a su casa cuando ya no es necesario. Regresar es una de las principales características del "buen migrante" incluso si se tiene en cuenta, como sabemos, que muchos programas de migración temporal no han logrado completar la última fase del proceso de migración: el retorno (por ejemplo, los programas de braceros entre Estados Unidos y México entre 1942 y 1964, los programas para trabajadores invitados entre Alemania y Turquía y otros países tras la Segunda Guerra Mundial). De este modo, la experiencia indica que el retorno no siempre se materializa como ilustra el "mito del retorno".

Aun así, no se ha aprendido nada de la experiencia anterior a la hora de crear políticas en materia de migración. La legislación actual de la UE sigue promoviendo distintos sistemas de trabajadores invitados, olvidando lo que ya se conoce del fracaso de dichos programas, como si la necesidad de traba- 
jadores específicos en los ciclos económicos de crecimiento justificara intentarlo una vez más. Para algunos expertos:

"Europa se está convirtiendo en una zona fronteriza. Esta transformación se caracteriza por una nueva categorización de espacios y territorios, una expansión y diversificación de las formas de control de fronteras y aplicación de la ley y un discurso público forjado por la distorsión en la descripción de los migrantes" (Euskirchen, Lebuhn y Ray, 2007)

Estos autores, citando a Tobias Pieper (2004), señalan que el sistema europeo de fronteras distingue cuatro tipos de personas según su valor económico para Europa: a) trabajadores altamente cualificados provenientes de la periferia que han sido reclutados o invitados a Europa; b) trabajadores invitados de escasa cualificación que llegan a Europa dentro de la legalidad con visados de corta duración para vivir y trabajar en unas condiciones muy restringidas; c) una mano de obra transnacional "ilegal" (sin papeles) que carece de todo derecho o protección para la negociación de sus condiciones de vida y de trabajo; y d) refugiados superfluos desde el punto de vista económico, a los que se les niega cada vez con mayor frecuencia cualquier tipo de estatus jurídico seguro en Europa (Euskirchen, Lebuhn y Ray, 2007).

La tarjeta azul de la UE (Directiva 2009/50/CE del Consejo, que entró en vigor el 19 de junio de 2009 y que tiene de plazo para su trasposición a los Estados miembros hasta el 19 de junio de 2011) es la última disposición legislativa de la UE que contempla, en teoría, la mejora de la capacidad de la Unión para atraer a trabajadores altamente cualificados de terceros países. Como los Estados miembros todavía están estudiando la directiva, hay muchas incertidumbres respecto a cómo se aplicará, aunque corre el riesgo de convertirse en otro programa más de migración temporal diseñado únicamente para inmigrantes internacionales titulados superiores y, por tanto, dirigido a "migrantes muy deseados y deseables" mientras no se conviertan en una carga para las sociedades europeas (los migrantes altamente cualificados perderán su tarjeta azul si se quedan en paro durante más de 3 meses o si solicitan asistencia social). De este modo, su deseabilidad está condicionada por su éxito económico en todas las circunstancias.

\section{LAS POLÍTICAS MIGRATORIAS: RESPUESTAS A LAS NECESIDADES DEL MERCADO LABORAL}

Desde el punto de vista de las políticas migratorias generales, existen políticas para promover la migración (ya sea la emigración o la inmigración) junto a políticas para retener a los futuros migrantes (evitar la migración mediante la creación de programas de desarrollo económico), políticas para regular los flujos, de recuperación (es decir, políticas de compromiso para traer de vuelta a 
los migrantes) y de integración, según los objetivos y propósitos de las políticas en cuestión. Por otro lado, otras tipologías clasifican las políticas migratorias en migración voluntaria o forzada (directiva de retorno), migración masiva (programas de colonización) o selectiva (programas de reclutamiento para trabajadores cualificados o no). En la mayoría de los casos, los mercados laborales y las oportunidades económicas repercuten de forma significativa en el fomento de determinados tipos de políticas, bien para atraer a migrantes o bien para favorecer su regreso, facilitando o impidiendo la movilidad. Basándonos en la propuesta de Mármora (2002), proponemos lo siguiente:

TABLA 1

Tipos de políticas migratorias

\begin{tabular}{l|l|l}
\hline Objetivos & Ejecución & Población destinataria \\
\hline $\begin{array}{l}\text { Promoción (emigración e inmigración) } \\
\text { Retención }\end{array}$ & $\begin{array}{l}\text { Voluntaria } \\
\text { Forzada }\end{array}$ & $\begin{array}{l}\text { Masiva } \\
\text { Selectiva (con y sin } \\
\text { cualificación }\end{array}$ \\
$\begin{array}{l}\text { Integración } \\
\text { Recuperación }\end{array}$ & & \\
\hline
\end{tabular}

Fuente: elaboración propia basada en Mármora 2002.

Actualmente, no son corrientes las políticas para fomentar las migraciones masivas, pese a que existen muchos casos de migración selectiva y algunos programas de reclutamiento o medidas de legalización pueden actuar como "llamada" a ese tipo de migración. A finales de la década de los noventa, Portugal se enfrentaba a una demanda en aumento de trabajadores para la construcción (Exposición Internacional de Lisboa en 1998, el nuevo puente sobre el Tajo, muchas autopistas en todo el país, los nuevos estadios para el Campeonato Europeo de Fútbol de 2004 y la burbuja de la construcción/inmobiliaria), que hizo que los empresarios realizaran una captación activa de trabajadores en países del Este de Europa no miembros de la UE y en Brasil en esa década, lo que generó más adelante un aumento exponencial de migrantes procedentes de esas regiones (véase la figura 2). En aquella época, como Portugal era un país de emigración, no contaba con muchas disposiciones legales o administrativas para tratar el flujo de inmigración, por lo que gestionar y controlar la inmigración resultó muy difícil.

Por consiguiente, el creciente número de personas que entraban y se instalaban en el país dio paso al caos y fue necesario aprobar nueva legislación para controlar y regular los flujos (decreto ley $\mathrm{n}^{\circ}$ 4/2001). A fin de resolver la situación, la ley tenía una doble vertiente, ya que aspiraba a regular las nuevas entradas al mismo tiempo que sentaba la base para regularizar a los que ya se encon- 
FIGURA 2

Evolución de la inmigración en Portugal desde 1980 y 2009 según la situación jurídica (permiso de residencia, de estancia y visado de larga duración)

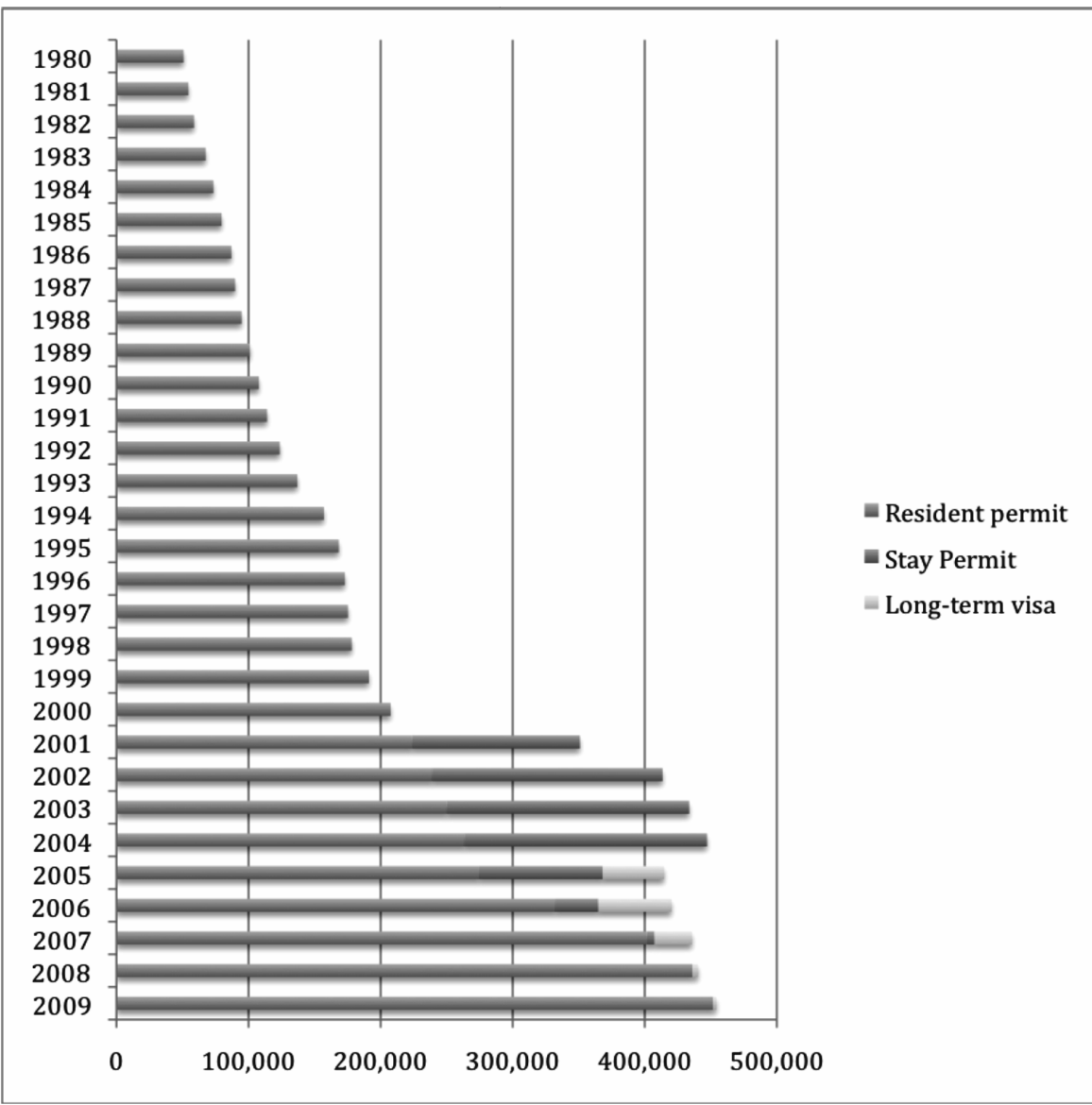

Fuente (Serviços de Estrangeiros e Fronteiras)

traban en el país con contratos laborales. No obstante, la disposición específica sobre la regularización conectaba dos aspectos diferentes: por un lado, permitía que los inmigrantes se establecieran de forma provisional y, por el otro, a la vez ligaba la permanencia a un contrato laboral renovable. Esa asociación, es decir, vincular la autorización a quedarse en el país de forma legal con disponer de un contrato de trabajo válido, es problemática y se ha convertido en uno de los problemas recurrentes del sistema, al impulsar un "círculo vicioso de irregularidad". 
En este sentido, la situación legal de los trabajadores inmigrantes depende de la voluntad del empresario de ofrecerles trabajos reales y no de la demanda real de trabajo, por lo que es normal que los migrantes pasen por periodos en los que están documentados y otros en los que no lo están (Padilla, 2007). Además, una vez se inicia el flujo migratorio, es difícil pararlo inmediatamente, sobre todo debido a la manera en que funcionan las redes sociales y a los procesos habituales de reagrupación familiar (Currant y Rivero-Fuentes, 2003; Padilla, 2006 y 2010). El gráfico 1 ilustra la evolución de los flujos de inmigración legal en Portugal y pone de relieve la diversidad de las situaciones jurídicas creadas en la última década.

Retomemos el tema de las políticas migratorias. Entre los programas más comunes para promover la migración se encuentran los que están dirigidos a poblaciones específicas, como: a) recursos humanos altamente cualificados, b) migración con capital, c) migración laboral y d) colonización. Es posible encontrar ejemplos de cada caso en los países de Latinoamérica y del Sur de Europa, principalmente España y Portugal, en los últimos años.

a) Recursos humanos altamente cualificados: por ejemplo, el acuerdo bilateral entre los países de origen (Uruguay y Cuba) y el de destino (Portugal) facilitó la llegada de 15 médicos/as uruguayos/as y más de 40 médicos/as cubanos/as. Además, la actual legislación sobre inmigración portuguesa incluye visados y permisos de residencia específicos para los que están altamente cualificados (con titulación universitaria) para trabajar en instituciones de investigación, intercambio de estudiantes, prácticas/formación y servicios voluntarios, y para los que trabajan en empresas multinacionales.

b) Migración con capital: cuando los inmigrantes traen dinero, normalmente no hay resistencia a que se instalen. Portugal, al igual que la mayoría de países, tiende a facilitar la llegada de migrantes/inversores con recursos financieros para abrir un negocio. La nueva ley sobre inmigración (Lei $\mathrm{n}^{\circ}$ 23/2007) incluye un tipo específico de visado a estos efectos, lo que demues-

\section{TABLA 2}

Art. 60 de la ley 23/2007 sobre inmigración

2- Se concederá un visado de residencia a aquellos inmigrantes emprendedores que deseen invertir en Portugal, siempre que:

a) hayan efectuado operaciones de inversión, o

b) demuestren contar con medios financieros existentes en Portugal, incluso un crédito financiero de instituciones financieras portuguesas, y demuestren, por cualquier medio, su intención de realizar operaciones de inversión en el territorio portugués. 
tra un cambio sustancial respecto a la legislación anterior que no contemplaba los permisos de residencia para los/as emprendedores/as. Al contrario, éstos estaban obligados a disponer de contratos como empleados dependientes para obtener el permiso de residencia.

c) Migración laboral: representa la situación más frecuente, si bien existen diversas modalidades e iniciativas para atraer la "mano de obra". La ley portuguesa incorpora un sistema de cupos basado tanto en las necesidades del mercado de trabajo (normalmente problemático y lento) como en una migración de prospección para que algunos migrantes cualificados vayan y busquen trabajo en el país. En España se solucionó la escasez de personas que se alistaban en el ejército con un programa que reclutaba a nacionales extranjeros, sobre todo latinoamericanos con pasaporte español, y que se amplió al reclutamiento de extranjeros, principalmente de Latinoamérica, con o sin antepasados españoles (véase la tabla con el anuncio).

\section{TABLA 3}

Anuncio de inscripción en las Fuerzas Armadas Españolas para extranjeros

Se abrió el período de inscripción anual para extranjeros dentro de las fuerzas armadas. La convocatoria está dirigida a hispanoamericanos o ecuatoguineanos y permite aprender una profesión a la vez que da el beneficio de un trabajo estable, con todos los beneficios sociales. Se lo promociona también por mejorar el grado de integración a la sociedad española. Esta convocatoria está abierta a los ciudadanos de: Argentina, Bolivia, Costa Rica, Colombia, Cuba, Chile, Ecuador, El Salvador, Guinea Ecuatorial, Guatemala, Honduras, México, Nicaragua, Panamá, Paraguay, Perú, República Dominicana, Uruguay o Venezuela.

Requisitos

- Poseer el Permiso de Residencia temporal o permanente o tener derecho reconocido por ley al acceso a las Fuerzas Armadas para extranjeros.

- Mayor de edad en la fecha de incorporación y no cumplir 27 durante el año. - Medir más de 1,55 m y menos de 2,03 m.

Compromiso: Hasta 6 años. Una vez cumplidos, si ha solicitado la nacionalidad española, tendrá la posibilidad de ampliarlo hasta un máximo de tres años. Cuando adquiera la nacionalidad española podrá firmar el compromiso de larga duración. Formación: Fase inicial de dos meses. Una vez superada firmará su compromiso como soldado. Destino: Se da la posibilidad de elegir entre la mayoría de los destinos y plazas disponibles. Salario inicial: Sueldo bruto en 2006 de 13.372,34 euros/año mínimo, incrementándose anualmente esta retribución. Alojamiento y vestuario: gratuito. Otros beneficios: Seguridad Social, Seguro de Vida y Accidentes, Seguro Médico, Otras ventajas sociales 
d) Programas específicos de colonización: se considera que las medidas para la colonización eran habituales en tiempos remotos y están asociadas a periodos de colonización, expansión y ocupación de tierras. No obstante, hace poco se han promovido en España y Portugal acciones específicas de colonización para repoblar con jóvenes aldeas cuya población está envejeciendo. Por lo general, esos programas dan preferencia a los ciudadanos con un origen étnico similar (percibido), es decir, los que ya poseen la nacionalidad del país de destino, con la esperanza de lograr un ajuste más fluido. Por ejemplo, en 2006, Vila de Rei (Portugal) firmó un acuerdo con Maringa, Paraná, en Brasil con el objeto de llevar familias de ascendencia portuguesa para que se asentaran en la localidad para contrarrestar la desertización y el envejecimiento de la población. España había hecho lo mismo en 2000, cuando la pequeña aldea de Aguaviva en Teruel anunció en Argentina 13 ofertas de empleo para aquellas familias numerosas con pasaporte español que repoblaran la zona (se recibieron más de cinco mil solicitudes). Las familias obtendrían trabajo y ayuda para la escolarización de sus hijos. El acuerdo incluía una cláusula que obligaba a los migrantes a quedarse un mínimo de 5 años. La comparación entre estos dos casos arroja similitudes. A) Los dos países de la península adoptaron medidas similares al elegir familias de ascendencia portuguesa y española. B) La población y los medios nacionales reaccionaron de forma semejante (los vecinos y los medios se referían a ellos como "los extranjeros", "inmigrantes", "brasileños/argentinos" y jamás "compatriotas portugueses/españoles").

Además, las dos iniciativas fracasaron desde el punto de vista de los recién llegados y de las autoridades locales. Los migrantes se desilusionaron porque no se habían cumplido las promesas ni sus expectativas y las autoridades locales se desanimaron por la divergencia entre la evaluación de la necesidad original y los criterios de selección. Por ejemplo, traer profesionales (altamente cualificados) o habitantes urbanos no eran buenas opciones para que se establecieran en una aldea rural en la que no podían utilizar sus conocimientos de forma adecuada. Sin embargo, habría que aprender la lección tras esas experiencias, incluso si sólo sirve para identificar una nueva tendencia en la elaboración de políticas migratorias. Como señala García (2007), esta tendencia representa la internalización o apropiación de las políticas sobre migración en el ámbito local y más casos de "glocalización".

\section{A QUÉ ATENERSE ANTE LA MIGRACIÓN CIRCULAR Y LA MIGRACIÓN ALTAMENTE CUALIFICADA. ALGUNAS EXPERIENCIAS DE PAÍSES LATINOAMERICANOS}

Pasemos ahora a la cuestión concreta de la migración circular. Aunque no se trata de un concepto nuevo, últimamente ha adquirido el interés y el apoyo de 
organizaciones internacionales (sobre todo de la OIM, la OCDE, la ONU), Estados y otras partes interesadas, no necesariamente los propios migrantes. En general, la migración circular se ha relacionado más estrechamente con la movilidad de los migrantes altamente cualificados, si bien no siempre es así, ya que los migrantes no cualificados (o de formación inferior) pueden regresar al país de origen llevando consigo innovación y otras prácticas. Sin embargo, nos centraremos en los trabajadores titulados o la movilidad internacional de talentos, utilizando la literatura latinoamericana y algunas de sus conclusiones.

Salt (2001) afirmó que la migración de los altamente cualificados "es hija de la globalización económica y las actividades de las compañías transnacionales". No obstante, esta afirmación sólo señala algunos aspectos de la movilidad actual. En Latinoamérica, la migración de los recursos humanos con titulaciones superiores constituye un problema endémico desde hace tiempo (Pellegrino y Martínez, 2001; Pellegrino, 2004 y 2001; Pellegrino y Calvo, 2001), aun cuando la investigación y la creación de políticas en el Norte no han reconocido ni se han preocupado adecuadamente de la situación hasta hace poco, como indican Barrere et. al.:

En la agenda de los países desarrollados y las organizaciones internacionales (en el caso de Sudamérica, destacan el Banco Mundial y el Fondo Monetario Internacional), las cuestiones consideradas prioritarias para el desarrollo de la región se refieren a las dimensiones comerciales y financieras, así como al comportamiento macroeconómico general de los distintos países, prestando escasa atención a los problemas que tratan de la formación y el suministro de mano de obra altamente cualificada. (2004: 6)

Tradicionalmente, la movilidad internacional de recursos humanos altamente cualificados en Latinoamérica estaba asociada a lo que se denominó el éxodo de intelectuales (en las décadas de los sesenta y setenta). Durante esa época, dos escuelas de pensamiento principales explicaron la llamada "fuga de cerebros": los internacionalistas y los nacionalistas. La primera escuela defendía la movilidad de los talentos/cerebros como si fuera una solución óptima y eficaz para el uso del talento, de forma que las personas que vivían/trabajaban en países que no eran productivos (subdesarrollados) ni contaban con los recursos para la investigación debían marcharse a países más eficientes para que, al final, el mundo se beneficiara de la dispersión de su talento y producción. Esta escuela cree en un bienestar mundial.

Por el contrario, la segunda postura, los nacionalistas, opina que los beneficios de la fuga de cerebros son unidireccionales y que normalmente los países ricos se beneficiaban más que los países pobres, porque los países de origen que habían invertido en la formación de esas personas con talento no 
percibían retribución o amortización alguna más adelante. Esta escuela cree también que la movilidad de los talentos no es tan libre, puesto que existen políticas activas de captación y, al final, el talento del Sur emigra al Norte. Podríamos emplear el ejemplo de algunos premios Nobel argentinos para ilustrar la filosofía de las dos escuelas: Bernardo Houssay (1947) y Luis Leloir (1970) se formaron en Argentina y trabajaban en ese país cuando fueron galardonados con el premio, mientras que César Milstein (1984) lo obtuvo en el Reino Unido, donde había migrado tras formarse en Argentina y tras haber logrado prestigio internacional en este último país.

Durante los años sesenta y setenta, se realizaron algunos estudios relevantes en Latinoamérica para comprender y explicar la migración de profesionales y trabajadores titulados, sobre todo porque muchos países latinoamericanos habían invertido o estaban invirtiendo en educación terciaria, lo que produjo la masificación de las universidades. Oteiza (1971) elaboró un modelo explicativo para la migración denominado el "diferencial de preferencia", que se compone de varios factores: a) el diferencial de ingresos, b) el diferencial de apoyo logístico, c) el diferencial de reconocimiento profesional (el prestigio de su trabajo), e incorpora un factor residual que incluye la situación política, el sistema universitario, junto con sus vías de promoción, y la discriminación ideológica, entre otros aspectos. Su estudio fue pionero, puesto que contribuyó a entender el panorama general de aquella época y todavía es de aplicación en la actualidad.

El reciente trabajo de Oteiza, "Cronología de una diáspora. Historia del brain drain argentino" (2000), ahonda en la cuestión y se remonta al pasado para explicar la llamada "fuga de cerebros", reflejando sus ideas al emplear este concepto. Divide la fuga de cerebros en cinco etapas. Su periodización empieza con el nacimiento de la república, dado que piensa que la historia de Argentina ha estado marcada por la movilidad de personas con talento, incluida la práctica de figuras y héroes nacionales de estudiar en el extranjero (en aquella época, sobre todo en Francia).

TABLA 4

Las cinco etapas de Oteiza en la fuga de cerebros de Argentina

Primera: 1810-1870

Segunda: 1870-1930

Tercera: 1930-1966

Cuarta: 1966-1983 (regímenes autoritarios, persecución ideológica)

Quinta: 1983-actualidad (globalización) 
Otros estudiosos se han centrado en la migración de médicos/as y demás talentos latinoamericanos, principalmente la diáspora científica. Recientemente se han producido ensayos (Solimano, 2006; Pellegrino, 2001; Barrere et al., 2004) que señalan que, incluso si se compara con otras regiones del mundo (Asia con China y la India, y África), la "pérdida" de Latinoamérica es residual, aunque, para la propia región, la migración de talento sea significativa.

En la actualidad, se ha abandonado el concepto de fuga de cerebros y se emplean otros términos en su lugar, como por ejemplo, la "circulación de talento internacional" y la "diáspora científica". En este sentido, las nuevas escuelas de pensamiento creen que es posible encontrar aspectos positivos en la movilidad internacional, no sólo para los individuos, sino también para los países de origen, sobre todo si se tiene en cuenta la situación de los países en desarrollo. De este modo, las migraciones pendulares o circulares, que implican retornos (temporales o permanentes), pueden contribuir al desarrollo o crecimiento de los países en desarrollo, por lo que se han acuñado expresiones como "ganancia", "intercambio" y "circulación de cerebros" para sustituir la "fuga de cerebros". Por ejemplo, los programas de formación, los intercambios o las prácticas de corta o larga duración pueden convertirse en una ventaja para los países de origen como instrumento de desarrollo. Otra expresión utilizada habitualmente es el "movimiento de capital humano" $(\mathrm{CH})$, que:

"puede identificarse, en la práctica, como el movimiento de científicos, ingenieros (por ejemplo, en el sector de la información), ejecutivos y otros profesionales a través de fronteras. Se trata de personas con talento especial, grandes competencias y conocimientos especializados en los ámbitos científico, tecnológico y cultural. Otra dimensión de la movilidad internacional del talento es la migración emprendedora, a saber, las personas con talento para la creación de negocios y la movilización de recursos en vez de personas que posean necesariamente un elevado capital en formación reglada" (Solimano, 2002: 7-8).

La literatura en el ámbito de la sociología y, más recientemente, en el de la economía, ha reconocido el importante efecto de red producido por la migración de talentos: la creación de redes empresariales y comerciales que constituyen una externalidad de la diáspora (Docquier y Rapoport, 2007). Este fenómeno también se ve reflejado en las diásporas científicas y esa externalidad es un elemento que pueden usar de forma positiva los países de origen. Por lo tanto, para determinar de qué manera la circulación de cerebros puede suponer una ventaja para esos países, es necesario conocer cuáles son esos talentos. Así, resulta práctico disponer de una taxonomía que enumera cuáles son. La siguiente tabla resume la clasificación de Solimano, que muestra un proceso de ajuste conceptual. 
TABLA 5

Taxonomías de los talentos intelectuales

\begin{tabular}{l|l}
\hline Solimano 2002 & Solimano 2006 \\
\hline 1. Movilidad del talento productivo: & 1. Talento técnico \\
emprendedores/as (incluidas las & 2. Científicos/as y académicos/as \\
diásporas étnicas), innovadores/as, & 3. Profesionales del sector sanitario: \\
expatriados/as & médicos y enfermeros \\
2. Movilidad del talento científico & 4. Emprendedores/as y \\
académico, incluidos los estudiantes & administradores/as \\
(diásporas científicas) & 5. Profesionales de organizaciones \\
3. Movilidad de los profesionales & internacionales \\
sanitarios y del talento cultural & 6. Talento cultural \\
\hline
\end{tabular}

Resulta interesante comprobar cómo la literatura actual no ve problema alguno en la movilidad internacional de esos talentos, pero hace una clara distinción con los/as médicos/as. Así, pese a que la lógica económica prevalece para explicar la movilidad de innovadores/as, ingenieros/as, empresarios/as, científicos/as, personal académico y talentos culturales, la lógica tras la migración de los profesionales sanitarios parece ser diferente. Se argumenta que se pone en peligro los sistemas sanitarios de los países en desarrollo cuando los médicos/enfermeros abandonan sus países durante periodos de escasez de personal sanitario. Sólo en ese caso, los intereses de los/as médicos/as y enfermeros/as pueden entrar en conflicto con los intereses del suministro de servicios sanitarios en el país de origen. No obstante, como señala Solimano (2006), la circulación de estos profesionales no siempre tiene lugar desde los países en desarrollo hacia los industrializados (Sur-Norte). Existen muchos casos de circulación Sur-Sur (Cuba ha enviado médicos/as a muchos países latinoamericanos y de otras regiones) para ayudar en crisis sanitarias o desastres naturales o para crear el sistema de salud nacional. Por último, la circulación de médicos/as y enfermeros/as entre países industrializados (Norte-Norte) es más común ahora.

En este sentido, algunas características específicas de los sistemas de salud y del sector sanitario condicionan el ejercicio de estos profesionales y originan una paradoja, que podría resumirse como sigue:

"Los profesionales sanitarios extranjeros suelen estar sujetos a requisitos de licencia. A menudo, se trata de procesos largos, complejos y costosos que, en la práctica, constituyen una barrera efectiva al acceso al mercado laboral local para los profesionales sanitarios extranjeros. Al mismo tiempo, debido 
a la escasez de profesionales de la salud en los países industriales, como Estados Unidos y Reino Unido, este colectivo tiene un acceso más fácil a visados de trabajo que otros profesionales". (Solimano, 2006: 4)

No obstante, teniendo en cuenta el conflicto moral que puede suponer la migración de profesionales sanitarios desde países en desarrollo hacia naciones avanzadas, siempre resulta fundamental ser precavido. Esta cuestión ha sido objeto de debate en la OMS durante mucho tiempo y no hay consenso sobre cómo resolverla. Para mitigar este tipo de migración se han propuesto algunos tipos de políticas: a) compensación (al país de origen, asistiendo o ayudando a sus sistemas sanitarios); b) reclutamiento ético (la OMS acaba de aprobar un código ético de reclutamiento para el ejercicio internacional); c) otra propuesta más controvertida, pero innovadora, viene de Noruega y propone pagar una tasa de transferencia al país de origen. La experiencia nos enseña que se trata de una cuestión muy delicada, en la que resulta muy difícil conciliar los intereses de todas las partes, puesto que los intereses de los países, los de los sistemas sanitarios y los de los profesionales del sector son más antagonistas que convergentes (Padilla y Pereira Miguel, 2009). Sin embargo, siempre es posible encontrar experiencias positivas de movilidad internacional y buenas prácticas (Padilla et al., 2009). Podría señalarse la excepcionalidad o diferenciación del sector sanitario respecto a los demás sectores de talentos internacionales, sobre todo debido a las implicaciones (éticas) de un sistema sanitario deficiente en el país de origen. Aun así, en cualquier caso, debemos preguntarnos hasta qué punto un país puede perder todos sus talentos (emprendedores/as, científicos/as, innovadores/as, etc.). Una respuesta rápida indica que no pueden sacrificarse los talentos en todos los ámbitos. Así, un término medio tendría que ser posible.

Algunas de las siguientes experiencias de migración circular muestran cómo casos distintos pueden tener mejores resultados que otros respecto a las implicaciones para los migrantes, al igual que para los países de origen y de destino.

a) El doctor X, de Mendoza, va a la capital de Argentina, Buenos Aires, para cursar la especialización (ortopedia pediátrica), donde aprende técnicas quirúrgicas únicas en el Hospital de Pediatría Garrahan. Más tarde, se marcha a Francia para especializarse más y allí aprende nuevas técnicas al mismo tiempo que enseña a sus colegas franceses las aprendidas en su país de origen. A continuación, regresa a Mendoza, su región natal, donde ejerce la medicina tanto en el sistema sanitario público como en el privado. Se trata de un ejemplo perfecto de circulación internacional de talento, con una situación en la que todos salen ganando. 
b) El doctor M, de Argelia, se marcha a Francia para trabajar en el Hospital Saint Antoine. Se reconoce su formación como médico pero la normativa actual sólo le permite ejercer en el sistema sanitario nacional y no le deja trabajar en el sector privado (donde los médicos nacionales ganan mucho dinero), imponiendo normas distintas para el desarrollo profesional de los médicos nacionales y el de los extranjeros. El resultado es una situación en la que Francia sale ganando y una combinación de ganancia y pérdida para el médico, que puede sentirse desanimado ante esta práctica de discriminación.

c) El doctor L, de Moldavia, llega a Portugal como migrante laboral para trabajar en el sector de la construcción y se le envía a un hospital para pintarlo. La paradoja estriba en que sufre un proceso de desaprovechamiento de su preparación pero está feliz de estar en un hospital. Después de todo, podría estar trabajando en otro lugar. Más adelante, se entera de un programa dirigido a reconocer las titulaciones de los médicos extranjeros. Aprueba el examen y puede volver a ejercer la medicina, pero sólo como médico generalista, sin que se aprovechen sus 20 años de experiencia en pediatría. Incluso si, en general, es una situación en la que todos ganan (Portugal necesita profesionales sanitarios y el doctor $\mathrm{L}$ puede ejercer de médico), la falta de reconocimiento de su especialización constituye un desperdicio de su formación, puesto que Portugal también necesita especialistas.

d) El doctor D, de Buenos Aires, llega a Lisboa para dar una conferencia. Presenta su trabajo sobre cateterismo (un procedimiento muy innovador que aprendió en la clínica bonaerense de Favaloro). Al final de su ponencia, se le acerca el propietario de una clínica en el País Vasco que quiere contratarlo. En menos de un mes, se traslada a España donde ejerce la medicina y enseña el nuevo procedimiento a sus colegas. Se ha producido una circulación de cerebro junto a una "transferencia tecnológica" hacia un país desarrollado, con el resultado de una situación en la que España gana y Argentina pierde. Sin embargo, puede haber una circulación con intercambio de cerebro en el futuro.

e) La doctora S, de Uruguay, llega a Portugal para trabajar en el Servicio de Emergencias (INEM) como parte de un acuerdo bilateral (es una de los 14 médicos uruguayos). No se le permite ejercer en el sector privado, pero incluso con una gran carga de trabajo y un sueldo medio, disfruta de la vida más que antes de su partida, cuando estaba en el paro. En su caso, se enfrenta a un futuro incierto, pues el contrato es de cinco años. No hay disposiciones para que se quede ni para que regrese a Uruguay. 
f) La doctora A, de La Plata (Argentina), es doctora en Química y realizó investigaciones de postdoctorado en Estados Unidos. Quiere regresar a Argentina, pero las oportunidades no abundan, hasta que se entera de un programa que pretende atraer de vuelta recursos humanos altamente cualificados, como científicos/as y académicos/as que se encuentran en la diáspora. Envía su solicitud y resulta seleccionada para el Programa Raíces. Además de repatriarse, puede regresar con nuevas conexiones de Estados Unidos y otras regiones del mundo, más conocimientos sobre financiación internacional y, lo que es más importante, asociaciones. En este caso ganan todos los países implicados (diseminación de redes científicas) y ella misma, dado que puede vivir en el lugar que ha escogido y contribuir a su país.

Las historias anteriores son verídicas y se pueden considerar positivas desde distintos puntos de vista, según la situación y los ojos del evaluador. Nos permiten extraer propuestas para recomendar políticas relativas a la circulación internacional de talentos:

a) Es deseable fomentar programas de intercambio que sean bidireccionales, en el sentido de que las dos sociedades/países se beneficien del intercambio. Estos programas son mejores porque se basan en el supuesto de que los dos socios/partes tienen algo que enseñar. Así, los países en desarrollo y las naciones avanzadas pueden tener algo con lo que contribuir. Cuando sea posible, es importante no suponer que el Norte enseña siempre, al contrario, debemos abogar por evitar las relaciones asimétricas. Cuanto más horizontales sean las relaciones, mejor.

b) Es esencial ofrecer igualdad de derechos y oportunidades al personal contratado para evitar la discriminación y las desigualdades en el sistema. Las prácticas de captación, contratación y promoción del talento internacional deben evitar ponerlos en situación de inferioridad, lo que iría en contra del principio por el que fueron captados y contratados en un primer momento. Las políticas deben prohibir las prácticas de tener médicos/as de primera y segunda clase. Asimismo, los programas deben prestar especial atención a las actitudes de los colegios/asociaciones profesionales, puesto que éstos poseen la facultad de facilitar o dificultar la incorporación de esos profesionales (médicos/as, enfermeros/as, arquitectos/as, etc.). Si no se tienen en cuenta esos aspectos, las buenas oportunidades percibidas en el país de origen pueden dar lugar a la desilusión, la frustración o expectativas no cumplidas en el país de destino.

c) En el caso específico de la movilidad de los profesionales sanitarios, resulta importante confeccionar programas de cooperación entre países del Norte y del Sur, así como de cooperación Norte-Norte y Sur-Sur. Estos programas deben tomar en consideración las necesidades del sistema sanitario 
del país de origen y los intereses de los propios profesionales de la salud (formación, promoción, reconocimiento, etc.).

d) La mayoría de los sistemas científicos, entre los que se encuentra el portugués, promocionan la internacionalización de las ciencias a través de la movilidad internacional. Este tipo de movilidad favorece la creación de diásporas científicas. En este sentido, la movilidad internacional debe intentar fomentar el intercambio, la formación, la circulación y las misiones científicas en ambas direcciones (origen y destino) garantizando que el talento "circula" y se genera diseminación en los dos sistemas.

e) Además de la circulación de cerebros, es importante promover la transferencia de tecnología y capital social para asegurar que también los países en desarrollo obtienen una parte justa de la circulación internacional de cerebros.

f) Los países de origen deben facilitar el retorno de las diásporas, tanto de emprendedores/as como de científicos/as, por lo que resulta fundamental que los países latinoamericanos conciban políticas para repatriar o recuperar parte de las diásporas o, cuando menos, para establecer conexiones que les permitan emplean las diásporas como una red de talentos. En este sentido, las políticas en el ámbito de la ciencia y la tecnología (para las diásporas científicas) son cruciales tanto para retener como para atraer de vuelta a la población con talento, ya que tienen un efecto multiplicador respecto a la creación de redes y respecto a los negocios.

g) Los países de origen y de destino deben emplear las diásporas existentes como "diplomáticos culturales" porque éstos pueden actuar como mediadores fomentando relaciones de intercambio entre sociedades. En general, tanto los países de destino como los de origen no utilizan plenamente a las diásporas, quizá porque ambos teman las lealtades nacionales. Las diásporas pueden ser útiles en diferentes etapas de la profesión, también si se traen a los migrantes tras su jubilación, cuando la gente desea volver a casa. Además, es importante darse cuenta de que las diásporas pueden contribuir al desarrollo de su país de origen sin retornar efectivamente.

h) Las políticas del país de origen o de destino deben evitar el desperdicio de cerebros o la falta de utilización plena de los recursos y talentos.

i) Resulta esencial no subestimar el deseo de contribuir que tiene la diáspora. Incluso si no hay muchas investigaciones sobre esta cuestión, a los/as expatriados/as, científicos/as, emprendedores/as y otros tipos de migrantes, en general, les gusta contribuir tanto al país de origen como al de destino, dado que se sienten en la obligación o el deber de ayudar. Los premios no financieros y el reconocimiento constituyen medios importantes para promover la colaboración entre países. Las diásporas son redes de cerebros que pueden contribuir al desarrollo de las dos sociedades. 


\section{BREVES OBSERVACIONES FINALES}

De los apuntes anteriores, se pueden extraer conclusiones sobre la movilidad de la migración altamente cualificada desde los países en desarrollo hacia los desarrollados. Una es el inmenso valor de las diásporas de talentos tanto para el país de origen como para el de destino, como interlocutores privilegiados para el diálogo con actores gubernamentales, además de como redes que pueden favorecer el desarrollo y el crecimiento directa e indirectamente en el origen y en el destino.

Actualmente existe una gran variedad de diásporas con talento, desde emprendedores/as y empresarios/as, técnicos/as y científicos/as, hasta profesionales sanitarios y expatriados/as, entre otros. Resulta más evidente que los Estados deben elaborar políticas y programas que mejoren su papel. Los países de origen pueden hacerlo fomentando políticas de compromiso que favorezcan el retorno cuando sea posible, junto con actividades de intercambio y cooperación que amplíen el alcance de la diáspora a otros grupos y generaciones. Además, las políticas de compromiso deben transcender el interés de las remesas y centrarse en facilitar la integración de los recién llegados. Los países de destino deben garantizar políticas justas para contratar, promocionar y ejercer la profesión a fin de evitar normas discriminatorias entre los talentos nacionales y los extranjeros, así como aplicar políticas de reclutamiento éticas que no pongan en peligro los intereses de los países de origen.

En un futuro cercano, la circulación de talentos será crucial para el crecimiento y la innovación en todo el planeta, de modo que derivar ventajas para los países de origen y de destino debe ser prioritario en las políticas de migración y cooperación internacional. A la hora de decidir qué tipos de políticas han de favorecerse, debe darse preferencia a las políticas sobre la "opción de la diáspora" antes que a las políticas sobre la "opción de retorno" debido a que las primeras producen una diseminación más completa y generosa.

\section{REFERENCIAS}

ACOSTA, DIEGO (2009). "The Good, the Bad and the Ugly in EU Migration Law: Is the European Parliament Becoming Bad and Ugly? (The Adoption of Directive 2008/15: The Returns Directive)" European Journal of Migration and Law, 11, 19-39.

BARRERE, R., L. LUCHILO y J. RAFFO (2004). "Highly Skilled Labour and International Mobility in South America", OECD Science, Technology and Industry Working Papers, 2004/10, OECD Publishing.

BROWN, PHILLIP (2001). 'Skill Formation in the Twenty-First Century', in Phillip Brown, Andy Green, and Hugh. Lauder, High Skills: Globalization, Competitiveness, and Skill Formation, Oxford: Oxford University Press: 1-55. 
CURRAN, SARA y RIVERO-FUENTES, ESTELA (2003) "Engendering Migrant Networks: The case of Miexican Migration", Demography, Volumen 40-Número 2, 289-307.

D'COSTA, ANTHONY (2006). "The International Mobility of Technical Talent. Trends and Development Implications", Research Paper $N^{\circ} 143$, United Nations University and World Institute for Development Economic Research (UNU-WIDER).

DOCQUIER, FRÉDERIC y RAPOPORT, HILLIEL (2004). "Skilled Migration: The Perspective of Sending Countries", Washington, DC: Banco Mundial.

EUSKIRCHEN, M. LEBUHN, H. y RAY, G. (2007). From borderline to borderland: The changing European border regime. In Monthly Review. Consultado el 19 de noviembre de 2010, en http://www.monthlyreview.org/1107euskirchen-lebuhn-ray.htm.

HIRST, PAUL y THOMPSON, GRAHANE (1996). Globalization in question. The international economy and the possibilities of governance. Cambridge: Polity Press.

MASANET, ÉRIKA y PADILLA, BEATRIZ (2010). "La inmigración brasileña en España y Portugal isistema migratorio ibérico?", Revista OBETS. Revista de Ciencias Sociales, Vol. 5 n. ${ }^{\circ}$ 1, pp. 49-86.

MEYER, JEAN-BAPTISTE y BROWN, MERCY (1999). "Scientific Diasporas: A New Approach to the Brain Drain". Discussion Paper No. 41, Management of Social Transformations (MOST).

MITTELMAN, JAMES (Ed.) (1997). Globalization: Critical Reflections, Boulder: Lynne Rienner Publishers.

NEDERVEEN PIETERSE, JAN (2003). "Social capital and migration. Beyond ethnic economies" Ethnicities, Vol. 3(1): 29-58.

OLSSON, ERIK (2009). "From exile to post-exile: the diasporisation of Swedish Chileans in historical contexts", Social Identities, 15: 5, $659-676$.

PADILLA, BEATRIZ (2010). "Migraciones Transatlánticas y Globalización: Brasileños en tierras lusas y el poder de las redes sociales", América Latina Hoy, 55, pp. 85-114.

PADILLA, BEATRIZ y ORTIZ, ALEJANDRA (2009). "Perfis sociais dos imigrantes latino-americanos em Portugal", Migrações $N^{\circ}$ 5, Observatório da Imigração, Lisboa, pp. 87-110.

PADILLA, BEATRIZ y PEREIRA MIGUEL, JOSÉ (2009). "Health and Migration in the EU: Building a shared vision for action". In Health and Migration in the EU: Better Health for All in and inclusive society, Parte I, editado por la Presidencia Portuguesa del Consejo de la UE. Pro-Book Publishing Limited: London, pp. 15-22.

PADILLA, BEATRIZ y PORTUGAL, RUI (Eds.) (2009). "Good Practices on Health and Migration in the European Union", in Health and Migration in the EU: Better Health for All in and inclusive society, Parte II, editado por la Presidencia Portuguesa del Consejo de la UE. Pro-Book Publishing Limited: London.

PADILLA, BEATRIZ (2007). "Acordos Bilaterais e Legalização: o impacte na integração dos brasileiros em Portugal" in A Imigração Brasileira em Portugal, editado por Jorge Malheiros. ACIME: Lisboa 
PADILLA, BEATRIZ (2006). "Brazilian Migration to Portugal: Social Networks and Ethnic Solidarity", CIES-ISCTE Documento de trabajo n. ${ }^{\circ}$ 12. http://cies.iscte.pt/ documents/CIES-WP12.pdf

Pellegrino, Adela (2004). "Migration from Latin America to Europe. Trends and Policy Challenges", Geneve, International Organisation for Migration, Research Series N. ${ }^{\circ} 16$.

PELLEGRINO, ADELA (2001). "Trends in Latin American Migration: Brain drain or Brain Exchange?" International Migration Vol 39 (5) Special Issue International Migration of the Highly Skilled.

PELLEGRINO, ADELA (2006). "La migración calificada en América Latina", artículo presentado en el Encuentro Iberoamericano sobre Migración y Desarrollo, Secretaría General Iberoamericana, Madrid, 18 y 19 de julio.

PELLEGRINO, ADELA, y CALVO, JUAN JOSÉ (2001). “¿Drenaje o éxodo?: Reflexiones sobre la migración calificada", Montevideo, Universidad de la República de Uruguay, Documento del Rectorado, 12 marzo.

PELLEGRINO, ADELA y MARTÍNEZ PIZARRO, JORGE (2001). "Una aproximación al diseño de políticas sobre migración internacional calificada en América Latina", CEPAL. Proyecto Regional de Población CELADE-FNUAP (Fondo de Población de las Naciones Unidas. Centro Latinoamericano y Caribeño de Demografía (CELADE) División de Población de la CEPAL. Santiago de Chile, Diciembre.

PELLEGRINO, ADELA y VIGORITO, ANDREA (2004). "Estrategias de sobrevivencia ante la crisis. Un estudio de la emigración uruguaya en 2002", Nordic Journal of Latin American and Caribbean Studies. Vol. XXXIV: 1-2. Estocolmo.

PELLEGRINO, ADELA y VIGORITO, ANDREA (2009). "La emigración calificada desde América Latina y las iniciativas nacionales de vinculación. Un análisis del caso uruguayo" Pensamiento Iberomamericano, Vol. 4 (1), pp. 189-215.

RAY, KRISTIN MICHELLE, LOWELL, LINDSAY y SPENCER, SARAH (2006). "International Health Worker Mobility: Causes, Consequences, and Best Practices", International Migration Vol. 44 (2).

RUÍZ SANDOVAL, ERIKA (2007). "Migración y desarrollo en América Latina: ¿Círculo vicioso o círculo virtuoso?" Pensamiento Iberomamericano, Vol. 0 (1), pp. 153-180.

SALT, JOHN (2001). Current Trends in International Migration in Europe, Council of Europe, CDMG, 2001, http://www.geog.ucl.ac.uk/mru/docs/current_trends_2001.pdf SASSEN, SASKIA (1998). Globalization and its Discontents. Essays on the New Mobility of People and Money, New York: New York Press, 1998.

SASSEN, SASKIA (1999). Guest and Alliens, New York: New Press, 1999.

SKELDON, RONALD (2009). "Of Skilled Migration, Brain Drains and Policy Responses", International Migration Vol. 47 (4).

SOLIMANO, ANDRÉS (Editor) (2008). The International Mobility of Talent: Types, Causes and Development Impact, Oxford: Oxford University Press.

SOLIMANO, ANDRÉS (2006a). "América Latina como fuente de talentos de nivel mundial: ¿se está rezagando la región?”, artículo presentado en el Encuentro 
Iberoamericano sobre Migración y Desarrollo, Secretaría General Iberoamericana, Madrid, 18 y 19 de julio.

SOLimano, Andrés (2006b) "The international mobility of talent and its impact on global development" Discussion Paper No8, United Nations University and World Institute for Development Economic Research (UNU-WIDER).

STRATTON, JON (2009). 'Uncertain lives: migration, the border and neoliberalism in Australia', Social Identities, 15: 5, 677 - 692

TIGAU, CAMELIA TIGAU (2010). "Latin American Scientific Diaspora: Before and After the Economic Recession", presentado en el Congreso de 2010 de la Latin American Studies Association, Toronto, Canadá, 6-9 de octubre.

VEGA, IVÁN (2003). "Emigración intelectual en Venezuela: el caso de la ciencia y la tecnología" INCI, Vol. 28, n. ${ }^{\circ}$ 5, pp. 259-267. 